\title{
A Novel Nonsense Mutation(c.1499C $>$ G) in CRB1 Caused Leber Congenital Amaurosis-8 in a Chinese Family and Literature Review
}

\author{
Wenhua Duan \\ Kunming Medical University \\ Taicheng Zhou \\ Affiliated Hospital of Yunnan University(The Second People's Hospital of Yunnan Province) \\ Huawei Jiang \\ Kunming Medical University \\ Minhui Zhang \\ DALI University \\ Min $\mathrm{Hu}$ \\ Affiliated Hospital of Yunnan University(The Second People's Hospital of Yunnan Province) \\ Liwei Zhang ( $\sim$ drzhangliwei@163.com ) \\ Affiliated Hospital of Yunnan University(The Second People's Hospital of Yunnan Province)
}

\section{Research Article}

Keywords: Leber's congenital amaurosis, Mutation, Crumbs homologue 1(CRB1)

Posted Date: February 17th, 2022

DOI: https://doi.org/10.21203/rs.3.rs-1281744/v1

License: @ (i) This work is licensed under a Creative Commons Attribution 4.0 International License. Read Full License 


\section{Abstract}

Background:Leber's congenital amaurosis (LCA) is a severe hereditary retinopathy disease that is characterized by early and severe reduction of vision,nystagmus,sluggish or absent pupillary responses. To date, the pathogenesis of LCA remains unclear,and the majority cases are caused by autosomal recessive inheritance. In this study, we explored the mutation in the Crumbs homologue 1(CRB1) gene in a Chinese family with LCA.

Methods:We conducted comprehensive ocular examinations and collected $5 \mathrm{ml}$ of blood samples from members of a Chinese family with LCA. The pathogenic gene was identified by capturing and sequencing the related genes of ocular diseases.

Results: We found a nonsense mutation(c.1499C>G) in the 6th exon of CRB1 in a Chinese family with LCA,which predicted a change of the protein p.S500X,may lead to loss of gene function.

Conclusions: This study reported a novel mutation(c.1499C>G,p.S500X) of the CRB1 gene occurred in a Chinese family with LCA, thus expanding the spectrum of CRB1 mutations causing LCA. And we summarize the 76 mutations reported so far in CRB1 that caused LCA8.

\section{Introduction}

Since Theodore Leber first described Leber's congenital amaurosis (LCA) 152 years ago(in 1869), We have obtained a great deal of information about LCA both in terms of clinical characteristics and molecular genetics.LCA, a rare but important juvenile retinal dystrophy, is an inherited retinal disorder most often diagnosed in infancy in the first 6 months of life and characterized by the presence of nystagmus, poor visual acuity (VA), and a severely reduced or nondetectable electroretinogram[1, 2]. In the worldwide,the prevalence of LCA is 1/81000 to $1 / 30000$ in newborn babies. Though the incidence is low, it also causes blindness in $20 \%$ of school-age children and accounts for approximately $5 \%$ of all hereditary retinopathy[3, 4]. LCA is currently described into 21 types according to the pathogenic genes, with autosomal recessive inheritance as the dominant. LCA8 is caused by homozygous or compound heterozygous mutation in the CRB1 gene (604210) on chromosome 1q31.

\section{Clinical Manifestation}

This study was performed in agreeent with the declaration of Helsinki. It was reviewed by the research unit's professional ethics committee and informed consent was obtained and signed by the investigator.

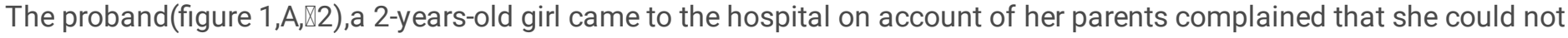
accurately grasp things. Her both eyes are performance as a horizontal pendulum nystagmus and was unable to comply with the detailed eye examination. Sequencing chromatograms:the proband show a homozygous mutation in CRB1 gene: nucleotide 1499 changed from cytosine $\mathrm{C}$ to guanine $\mathrm{G}$ (c.1499C囚G) homozygous mutation(figure 1,B) .Under the guidance of the paediatrician, the opportunity for examination was obtained through oral anesthesia.On examination, her eyes are in normal position, the cornea and lens are clear, fundoscopy showed the color of the optic disc in both eyes was light, and the blood vessels from both eyes were thin and narrow(figure 1,C). The pigmentation of the retina at the posterior pole was peppery and salt-like, and the macular area was a mass of lesions with a lot of pigmentation. Her parents and sister underwent detailed eye examinations(the results show in Table 1), including binocular corrected visual acuity, slit lamp examination, fundus photography, macular and optic disc OCT scanning, electroretinogram(ERG),which showed normal results(figure 1,D).

Table 1. Clinical examination data 


\begin{tabular}{|c|c|c|c|c|c|c|c|c|c|c|}
\hline \multirow{2}{*}{ Patient } & \multirow{2}{*}{ Gender } & \multirow{2}{*}{ Age } & \multirow{2}{*}{ Substitution } & \multicolumn{2}{|l|}{ UCVA } & \multicolumn{2}{|l|}{ CVA } & \multirow{2}{*}{$\begin{array}{l}\text { Corneal } \\
\text { optical } \\
\text { refection }\end{array}$} & \multirow[t]{2}{*}{ Nystagmus } & \multirow{2}{*}{$\begin{array}{l}\text { Globe } \\
\text { Retraction }\end{array}$} \\
\hline & & & & OD & OS & OD & OS & & & \\
\hline 邓:5 & $M$ & 34 & & 1.0 & 1.0 & 1.0 & 1.0 & Normal & - & - \\
\hline 邓:8 & $\mathrm{F}$ & 28 & & 0.6 & 0.7 & 1.0 & 1.0 & Normal & - & - \\
\hline$\nabla: 1$ & $\mathrm{~F}$ & 5 & & 0.6 & 0.6 & 0.8 & 0.8 & Normal & - & - \\
\hline $\mathbb{\nabla}: 2$ & $\mathrm{~F}$ & 2 & p.S500X & unable & unable & unable & unable & Normal & + & - \\
\hline
\end{tabular}

Features of LCA8 and unafected relatives. UCVA uncorrected visual acuity, CVA corrected visual acuity, LCA Leber's Congenital amaurosls. OD right eye, OS lef eye.

For LCA, the criteria are: signs of blindness or severe visual impairment from birth or within the first year of life,an ERG reduction of more than $50 \%$, and congenital nystagmus[5].Fundus examinations could reveal diagnostic clues, including peripheral pigmentary retinopathy, central maculopathy with or without bull's eye pattern, or even macular atrophy. And, indispensable, molecular confirmation is needed.

In our study,the proband's eye examinations and genetic tests were consistent with the diagnosis of LCA. The homozygous mutation in the 6th exon of CRB1: nucleotide 1499 changed from cytosine $C$ to guanine $G$ (c.1499C凶G), resulting in nonsense mutation of amino acids (p.S500X) which hasn't been reported before. Through genealogical analysis,the proband's parents and sister had heterozygous variation at this site.According to the ACMG(American College of Medical Genetics and Genomics) guidelines, the mutation was preliminarily determined to be pathogenic:PVS1+PM2+PM3_Supporting(hom). PVS1:This mutation is a zero-effect mutation (nonsense mutation), which may lead to loss of gene function; PM2: The frequency in the database of normal population is -, which is low-frequency variation; PM3_Supporting(HOM) : This mutation is a homozygous rare variant. No correlation of this locus was reported in the literature database. No pathogenicity analysis results were found in ClinVar database. Our study expanding the spectrum of CRB1 mutations causing LCA.

We use ScanProsite tool (https://prosite.expasy.org/scanprosite/) to check the secondary structure of CRB1 protein,the nonsense mutation(c.1499C>G,p.S500X) is in Laminin G domain profile 485-670:score $=32.931$. L, Yang,et.al. also reported a nonsense mutation(c.1576C>T,p.R526X) in this domain[6].The Laminin $G$ is an around 180 amino acid long domain found in a large and diverse set of extracellular proteins. It often occures in multiple copies probably serving as general protein interaction domains that bind the target proteins and other macromolecules, such as carbohydrates. In most proteins, the precise function of the laminin $\mathrm{G}$ domain is unknown. A large number of ligands in the $\mathrm{G}$ domain of laminin has been reported, including heparin, sulfatides, integrins, dystroglycan, nidogen, and fibulin.In neurexin the $\mathrm{G}$ domain is known to bind neurexophilins, a-latrotoxin and neuroligins $[7,8]$.

Another anatomical feature of LCA includes decreased thickness in different layers, especially in the outer nuclear layer (ONL), loss of integrity in the ellipsoid zone, and disorganized macular atrophy[9]. Unfortunately, the proband we reported was too young to cooperate with optical coherence tomography (OCT) and ERG examination, so we could not analysis the clinical features of these two aspects.

\section{Lca Caused By Crb1}

In 2004, Hanein,S.,et al.[10]reported a comprehensive mutational analysis of the all known genes in 179 unrelated LCA patients, including 52 familial and 127 sporadic cases. The result showed that mutations were identified in $47.5 \%$ patients.GUCY2D appeared to account for most LCA cases of our series(21.2\%), followed by CRB1 (10\%), RPE65 (6.1\%), RPGRIP1 (4.5\%), AIPL1 (3.4\%), TULP1 (1.7\%), andCRX (0.6\%).Three years later,Francesca Simonelli,et al.[11] analyzed 95 patients in Italian with LCA.They identified some novel variants which occurred more frequently in the in the RPE65 
(8.4\%), CRB1 (7.4\%),and GUCY2D (5.2\%) genes. Through a detailed ophthalmic evaluation of patients with the mutation,they found that CRB1 mutations were associated with reduced retinal thickness and a coarsely laminated retina(by OCT).In London,Henderson,R.H.,et.al. acquired DNA samples from 250 probands with LCA/early-childhood-onset retinal dystrophy (EORD).They analysed using the LCA chip and twenty-one probands were found to have mutations in CRB1[12].Corton,M,et.al. enrolled 404 Spanish cases in study, 114 of which suffered from LCA and 290 from EORP(early-onset RP). Their study revealed that $11 \%$ of Spanish patients carried mutations in CRB1, ranging from $9 \%$ of EORP to $14 \%$ of LCA cases. And more than three quarters of the mutations identified have been first described in their study[13].

Liping Yang et,al[6] through 18cases presenting with LCA to identify disease-causing mutations.They report compound heterozygous mutations of the CRB1 gene which included three novel heterozygous mutations: c.3059delT (p.M1020SfsX1), c.3460T>A (p.C1154S), and c.4207G>C (p.E1403Q). Hosono, K., et al reports the mutations of LCA and inherited retinal dystrophy(IRD) associated genes in 34 Japanese families, which is the frst to conduct a next generation sequencing(NGS) based molecular diagnosis of a large Japanese LCA cohort, achieved a detection rate of approximately $56 \%$. Their results show that the most frequently mutated genes were CRB1, NMNAT1, and RPGRIP1[14].In recently, Zhu, L., et al.[15]enrolled 37 patients with strictly defined LCA in a cohort of IRD in ten years(2009-2019). Their results revealed that CRB1 gene occupied a greater proportion(27\%) associated LCA in the western Chinese population.

CRB1 mutation is a common cause of LCA, and related mutations include missense mutation, nonsense mutation, insertion, deletion and splicing. The following Table 2 lists the mutations in LCA caused by CRB1 which including mutation types, sites,corresponding amino acid changes and regions in recent years. These results are for readers' verification and reference.

Table 2.Summary of CRB1 mutations caused LCA8

Exon Mutation type DNA change Amino acid change Region Reference

Ex1 Missense 2T囚C M1T Japanese Hosono, et al [14]

Ex1 Splicing $70+2 T>A$ Aberrant splicing Chinese Zhu, L., et al.[15]

Ex2 Nonsense 107C $\mathbb{G} \quad$ S36X Pakistan McKibbin,M.,e,a[16]

Ex2 Nonsense 424G囚T G142X uncertain Beryozkin A,et.al.[17]

Ex2 Nonsense 471C >A C157X Chinese Zhu, L., et al.[15]

Ex2 Insertion 481dupg A161G fs*8 Spanish Corton,M,et.al. [13]

Ex2 Deletion 498_506del9 I167_G169del England Ahmed, S,et.al. [18]

Ex2 Deletion 613_619del I205D fs*13 Spanish Corton,M,et.al. [13]

Ex2 Missense 614T囚C I205T England Henderson,et.al. [12]

Ex3 Missense $664 \mathrm{G}>\mathrm{A}$ E222K Chinese Li, L., et.al.[19]

Ex3 Insertion 668dupT L223Ffs*4 Japanese Hosono, et.al [14]

Ex3 Insertion 733dupg A245Gfs*16 Japanese Hosono, et al [14]

Ex3 Missense $866 \mathrm{C} \llbracket \mathrm{T} \quad$ T289M Italian Simonelli,et al.[11]

Ex3 Missense 998G > A G333D Korea Moon,S, e.a.[20]

Ex6 Deletion 1334_1740del C445Yfs*8 Japanese Hosono, et al [14] 


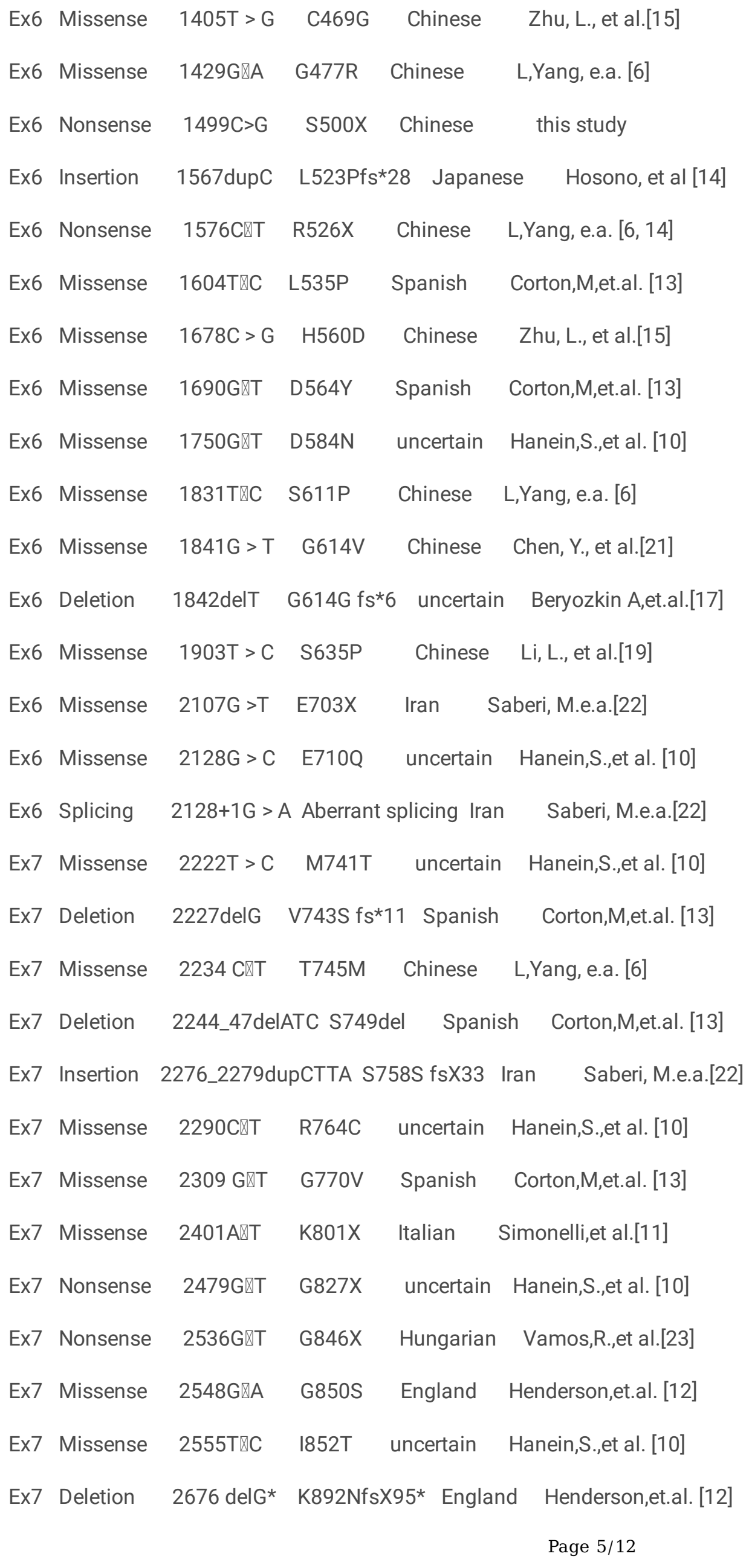


Ex8 Splicing 2677-2A囚C Aberrant splicing Chinese Lin Li, e.a.,[24]

Ex8 Deletion 2678-2682del5bpCCAAC S893S fs*14 uncertain Beryozkin A,et.al.[17]

Ex8 Nonsense 2688T $₫ A \quad$ C896X Spanish Corton,M,et.al. [13]

Ex8 Missense 2714G > A R905Q Chinese Zhu, L., et al.[15]

Ex9 Missense 2843G囚A C948Y Polish Skorczyk, et al [25]

Ex9 Missense 2843G $₫ \mathrm{~T} \quad \mathrm{C} 948 \mathrm{~F}$ uncertain Hanein,S.,et al. [10]

Ex9 Splicing 2853_2854insT A 952fsX972 uncertain Hanein,S.,et al. [10]

Ex9 Missense 2945C > A T982K Chinese Zhu, L., et al.[15]

Ex9 Missense $3002 \mathrm{~T} \otimes \mathrm{A} \quad$ I1001N Spanish Corton,M,et.al. [13]

Ex9 Missense $\quad 3017 \mathrm{C}>\mathrm{A} \quad$ S1006Y Chinese $\quad$ Zhu, L., et al.[15]

Ex9 Missense $\quad$ 3023T > G L1008X Chinese Zhu, L., et al.[15]

Ex9 Deletion 3059delT M1020SfsX1 Chinese L,Yang, e.a. [6]

Ex9 Missense 3068TهG L1023R Japanese Hosono, et al [14]

Ex9 Missense 3074GهT S1025I uncertain Hanein,S.,et al. [10]

Ex9 Nonsense $3152 \mathrm{G} \triangle \mathrm{A} \quad$ W1051X Spanish Corton,M,et.al. [13]

Ex9 Missense $3218 T$ > A L1073Q Chinese Zhu, L., et al.[15]

Ex9 Missense 3221T囚C L1074S Chinese Lin Li, e.a.,[16]

Ex9 Missense 3290T > A L1097Q Chinese Zhu, L., et al.[15]

Ex9 Missense 3299 T囚C I1100T Spanish Corton,M,et.al. [13]

Ex9 Missense $3307 \mathrm{G} \llbracket \mathrm{A} \quad$ G1103R Italian Simonelli,et al.[11]

Ex9 Missense 3320T囚G L1107R uncertain Hanein,S.,et al. [10]

Ex9 Deletion 3345delT G1115fsX1140 uncertain Hanein,S.,et al. [10]

Ex9 Missense 3466G囚T D1156Y uncertain AI Hollander,e.a.[26]

Ex9 Missense 3482A囚G Y1161C Spanish Corton,M,et.al. [13]

Ex9 Insertion 3542dupg C1181WfsX12* England Henderson,et.al. [12]

Ex11 Nonsense $3879 G \llbracket A \quad$ W1293X uncertain Hanein,S.,et al. [10]

Ex11 Missense 3961T®A C1321G uncertain Hanein,S.,et al. [10]

Ex11 Deletion 3988delG E1330fsX1340 uncertain Hanein,S.,et al. [10]

Ex11 Deletion 4000delG V1334W fs*7 Spanish Corton,M,et.al. [13] 

Ex12 Splicing
$4005+1 G>$ A Aberrant splicing Chinese
Zhu, L., et al.[15]
Ex12 Splicing
4013+1 G囚T Aberrant splicing uncertain
A I Hollander,e.a.[21]
Ex12 Deletion
4121_4130del R1374fsX1397 uncertain
Hanein,S.,et al. [10]

Table 3.Types and proportion of CRB1 mutations caused LCA8

\begin{tabular}{|llllll|}
\hline Types of mutations & Missense & Deletion & Nonsense & Insertion & Splicing \\
\hline count & 41 & 13 & 10 & 6 & 6 \\
\hline percentage & $53.9 \%$ & $17.1 \%$ & $13.2 \%$ & $7.9 \%$ & $7.9 \%$ \\
\hline
\end{tabular}

Table 4.Numbers and proportion of CRB1 exon mutations caused LCA8

\begin{tabular}{|lllllllllllll|}
\hline exon & Ex1 & Ex2 & Ex3 & Ex4 & Ex5 & Ex6 & Ex7 & Ex8 & Ex9 & Ex10 & Ex11 & Ex12 \\
\hline count & 2 & 7 & 5 & 0 & 0 & 17 & 13 & 4 & 21 & 0 & 4 & 3 \\
\hline percentage & $2.6 \%$ & $9.2 \%$ & $6.6 \%$ & 0 & 0 & $22.4 \%$ & $17.1 \%$ & $5.3 \%$ & $27.6 \%$ & 0 & $5.3 \%$ & $3.9 \%$ \\
\hline
\end{tabular}

Up to now, a total of 76 certain CRB1 mutations caused LCA.Furthermore, it has been reported that mutations in the CRB1 are responsible for $7.4 \%-27 \%$ of LCA in different populations. The pathogenic mutations were mainly missense, deletion and nonsense mutations, including insertion and splicing, which reflected the richness of mutation types(Table 3 ). The mutation sites of LCA8 were mainly concentrated in exon 6, 7 and 9 of CRB1, and the clear pathogenic sites were found in all exons except exon 4, 5 and 10, indicating the universality of mutation regions(Table 4). The reported cases involved more than 10 countries and regions, including China, England, Japan, Spain and Italy, which also showed that the global coverage of LCA caused by CRB1 is extensive.

\section{Other diseases of retinal dystrophy caused by CRB1 mutations:}

In addition to LCA,mutations in CRB1 are associated with several other diseases of retinal dystrophy:Rosa Riveiro-Alvarez, et.al.[27] reported early-onset RP phenotype Spanish family which was caused by the CRB1 p.Cys948Tyr (c.2843G>A) mutation.Two CRB1 missense mutations, c.C3991T:p.R1331C and c.C4142T:p.P1381L, were reported illustrate a novel presentation of a macular dystrophy caused by CRB1 mutations by Stephen H. Tsang et,al.[28].Arif O. Khan et,al uncovered a homozygous CRB1 mutation (c.80G $\triangle \mathrm{T}$ [p.Cys27Phe]) in three siblings with childhood cone-rod dystrophy and macular cystic degeneration in a family[29].Ajoy Vincent et,al reported biallelic mutations (p.Gly123Cys and p.Cys948Tyr, p.lle167_Gly169del and p.Arg764Cys) in CRB1 in two families caused autosomal recessive Familial Foveal Retinoschisis, which maybe the mildest end of the spectrum of CRB1-related diseases[30].Benjamin K. Ghiam et,al reported a novel mutation (c.4014T > A)in CRB1 was related with retinal degeneration and may portend a poor prognosis for CME responsiveness to therapy[31].

\section{Discussion}

LCA is the earliest and most severe hereditary retinopathy, in which the function of cone-rod cells in both eyes is completely lost at birth or within one year after birth, leading to congenital blindness in infants. The majority cases are caused by autosomal recessive inheritance.Typical characteristics of LCA includes:early and severe reduction of vision associated with 
nystagmus,photophobia, sluggish or absent pupillary responses,finger pressure on eyeballs;fundus appearance, ranging from normal, maculopathy, to typical RP-like abnormalities; and electroretinogram showed that A and B waves were flat and even severely reduced to non-detectable. It also can be accompanied by keratoconus, hyperopia, developmental delay and nervous system abnormalities et.al.[32]

In some cases/reports,there are many similar clinical features between LCA and early-onset RP and even the diagnosis is ambiguous[33].Early-onset RP,usually, is considered as a relatively milder form,which patients do not have a congenital onset of visual impairment. We could distinguish the following phenotypes: LCA,early onset retinal degeneration;RP, presence of preservation of the para-arteriolar retinal pigment epithelium and Coats-like vasculopathy[34].

So far,21 pathogenic genes associated with LCA have been reported. CRB1 belongs to LCA8.CRB1 gene maps to chromosome 1q31.3,is composed of 12 exons,the longest isoform consists of 1,406 amino acids. This gene encodes a protein which is similar to the Drosophila crumbs protein and localizes to the inner segment of mammalian photoreceptors. In Drosophila crumbs localizes to the stalk of the fly photoreceptor and may be a component of the molecular scaffold that controls proper development of polarity in the eye[35],and CRB1 has been found to be important in maintaining cellular polarity[36].

In the mouse retina, CRB1 is expressed in the inner segment of the photoreceptors and Muller cells to maintain adequate morphogenesis and polarity in retinal development[37]. Therefore, CRB1 gene mutations often lead to a variety of retinal dystrophy, including retinitis pigmentosa (RP), LCA ,macular dystrophy and so on. Approximately $9-17 \%$ of LCA cases have been related to CRB1 mutations, especially which are higher in the Chinese population[38, 39].A wide variety of visual acuity was noted in patients with mutations in CRB1,ranging from 20/30 to NLP[10,40].

Among LCA,RPE65 mutations were almost always associated with normal macular thickness, as assessed by OCT, whereas CRB1 mutations were associated with reduced retinal thickness and a coarsely laminated retina.Fundus abnormalities were more heterogeneous in carriers of CRB1 mutations. In fact, Some scholars observed salt-and-pepper retinal dystrophy in younger patients and subsequently massive spicular and not nummular pigmentation at the posterior pole, which was reported to be a phenotypic feature of carriers of CRB1 mutations[11].Saloni Walia et.al.[41] through a multicentere retrospective observational study with 169 patients of LCA found that mutations in RPE65(LCA-Type li) and CRB1(LCA-8) may be associated with a relatively better VA in early life compared with other gene mutations. And, onset of the symptoms of LCA after the age of 1 year is also associated with an overall better VA prognosis.

\section{Conclusions}

LCA is one of the earliest and most severe forms of inherited IRD, the patients suffer from severe visual impairment during childhood, with their vision continuously deteriorating, the final outcome of which usually is complete loss of vision by their thirties or forties[42].Therefore, it is very important to find an effective treatment. Albert $\mathrm{M}$ et,al. provided an entirely new dimension in ocular therapeutics for gene-therapy to LCA2, patients with LCA2 who received AAV2.hRPE65v2 by subretinal injection showed evidence of improvement in retinal function,in the pupillary light reflex, reduction in nystagmus. This clinicaltrials approaches to the treatment of LCA and possibly other forms of retinal degeneration[43].

Although much is still unknown about the pathogenesis of LCA. However, with the improvement of next-generation sequencing technology and the application of various molecular biological means, the research on corresponding cell functions, the identification of gene subtypes and the establishment of animal models have greatly promoted our understanding of LCA. These latest advances provide a steady stream of evidence for a better understanding and treatment of LCA in the future. And may be useful for faster gene diagnosis, prenatal testing, the development of potential gene therapies, and for improving the understanding of the molecular pathogenesis of LCA.

\section{Declarations}

\section{Ethics approval and consent to participate}

Page $8 / 12$ 
This study was approved by the Ethics Committee of Affiliated Hospital of Yunnan University. All experimental protocols were approved by the Affiliated Hospital of Yunnan University,and methods were carried out in accordance with relevant guidelines and regulations.All participants were informed about the purpose of the protocol and signed consent forms. The guardian(parent) of the patients consented to participation of the study.

\section{Consent to publish}

Written informed consent was obtained from the guardian(parent) of the patients,and they consented to publication of the study. The guardian (parent) of the patients consented for their medical information to be published.

\section{Availability of data and materials}

The relevant data were generated during this study and included in this article. And raw sequence data were not applicable to share in this article as no datasets were generated during the current study. The corresponding author Liwei Zhang (drzhangliwei@163.com) should be contacted if someone wants to request the data from this study.

\section{Competing interests}

The authors declare that they have no competing interests.

\section{Funding}

This study was supported by the National Natural Science Foundation of China (81860171),Medical Reserve Talents of Yunnan Province (H-2018020),Leading the charge of Yunnan Province Health System(L-2018018).

\section{Acknowledgments}

The authors thank the patients and all family members for their participation in this study.

\section{Authors Contribution}

Wenhua Duan and Taicheng Zhou carried out the experiments,and drafted the manuscript. Huawei Jiang and Minhui Zhang prepared the figure and tables. Liwei Zhang and Min Hu designed and funded this study.All authors read and approved the final manuscript. Dr.Min Hu(fudanhumin123@sina.com) and Dr. Liwei Zhang (drzhangliwei@163.com) are co-corresponding authors for this paper.

\section{References}

1. Koenekoop RK. An overview of Leber congenital amaurosis: a model to understand human retinal development. Surv Ophthalmol 2004; 49(4):379-98.

2. Traboulsi El, Koenekoop R, Stone EM. Lumpers or splitters? The role of molecular diagnosis in Leber congenital amaurosis. Ophthalmic Genet 2006; 27(4):113-5.

3. Koenekoop RK, Lopez I, den Hollander Al, Allikmets R, Cremers FP. Genetic testing for retinal dystrophies and dysfunctions: benefits, dilemmas and solutions. Clin Exp Ophthalmol 2007; 35(5):473-85.

4. Chen TC, Huang DS, Lin CW, Yang CH, Yang CM, Wang VY, Lin JW, Luo AC, Hu FR, Chen PL. Genetic characteristics and epidemiology of inherited retinal degeneration in Taiwan. NPJ Genom Med 2021; 6(1):16.

5. Booij JC, Florijn RJ, ten Brink JB, Loves W, Meire F, van Schooneveld MJ, de Jong PT, Bergen AA. Identification of mutations in the AIPL1, CRB1, GUCY2D, RPE65, and RPGRIP1 genes in patients with juvenile retinitis pigmentosa. J Med Genet 2005; 42(11):e67.

6. Yang L, Wu L, Yin X, Chen N, Li G, Ma Z. Novel mutations of CRB1 in Chinese families presenting with retinal dystrophies. Molecular Vision 2014; 20:359-67. 
7. Sung U, O'Rear JJ, Yurchenco PD. Localization of heparin binding activity in recombinant laminin $\mathrm{G}$ domain. EurJBiochem 1997; 250(1):138-43.

8. Beckmann G, Hanke J, Bork P, Reich JG. Merging Extracellular Domains: Fold Prediction for Laminin G-like and Aminoterminal Thrombospondinlike Modules Based on Homology to Pentraxins. J Mol Biol 1998; 275(5):725-30.

9. Cideciyan AV, Jacobson SG. Leber Congenital Amaurosis (LCA): Potential for Improvement of Vision. Invest Ophthalmol Vis Sci 2019; 60(5):1680-95.

10. Hanein S, Perrault I, Gerber S, Tanguy G, Barbet F, Ducroq D, Calvas P, Dollfus H, Hamel C, Lopponen T, Munier F, Santos L, Shalev S, Zafeiriou D, Dufier J-L, Munnich A, Rozet J-M, Kaplan J. Leber congenital amaurosis: Comprehensive survey of the genetic heterogeneity, refinement of the clinical definition, and genotype-phenotype correlations as a strategy for molecular diagnosis. Human Mutation 2004; 23(4):306-17.

11. Simonelli F, Ziviello C, Testa F, Rossi S, Fazzi E, Bianchi PE, Fossarello M, Signorini S, Bertone C, Galantuomo S, Brancati F, Valente EM, Ciccodicola A, Rinaldi E, Auricchio A, Banfi S. Clinical and molecular genetics of Leber's congenital amaurosis: a multicenter study of Italian patients. Invest Ophthalmol Vis Sci 2007; 48(9):4284-90.

12. Henderson RH, Mackay DS, Li Z, Moradi P, Sergouniotis P, Russell-Eggitt I, Thompson DA, Robson AG, Holder GE, Webster AR, Moore AT. Phenotypic variability in patients with retinal dystrophies due to mutations in CRB1. Br J Ophthalmol 2011; 95(6):811-7.

13. Corton M, et.al. High frequency of CRB1 mutations as cause of Early-Onset Retinal Dystrophies in the Spanish population. Orphanet J Rare Dis 2013; 8:20.

14. Hosono K, Nishina S, Yokoi T, Katagiri S, Saitsu H, Kurata K, Miyamichi D, Hikoya A, Mizobuchi K, Nakano T, Minoshima S, Fukami M, Kondo H, Sato M, Hayashi T, Azuma N, Hotta Y. Molecular Diagnosis of 34 Japanese Families with Leber Congenital Amaurosis Using Targeted Next Generation Sequencing. Sci Rep 2018; 8(1):8279.

15. Zhu L, Ouyang W, Zhang M, Wang H, Li S, Meng X, Yin ZQ. Molecular genetics with clinical characteristics of Leber congenital amaurosis in the Han population of western China. Ophthalmic Genet 2021; 42(4):392-401.

16. McKibbin M, Ali M, Mohamed MD, Booth AP, Bishop F, Pal B, Springell K, Raashid Y, Jafri H, Inglehearn CF. GenotypePhenotype Correlation for Leber Congenital Amaurosis in Northern Pakistan. Arch Ophthalmol 2010; 128(1):107-13.

17. Beryozkin A, Zelinger L, Bandah-Rozenfeld D, Harel A, Strom TA, Merin S, Chowers I, Banin E, Sharon D. Mutations in CRB1 are a Relatively Common Cause of Autosomal Recessive Early-Onset Retinal Degeneration in the Israeli and Palestinian Populations. Invest Ophthalmol Vis Sci 2013; 54:2068-75.

18. Ahmed Khan S, Richard Nestel A. CRB1 Gene Mutation Causing Different Phenotypes of Leber Congenital Amaurosis in Siblings. J Ophthalmic Vis Res 2019; 14(4):518-24.

19. Li L, Xiao X, Li S, Jia X, Wang P, Guo X, Jiao X, Zhang Q, Hejtmancik JF. Detection of variants in 15 genes in 87 unrelated Chinese patients with Leber congenital amaurosis. PLoS One 2011; 6(5):e19458.

20. Seong MW, Kim SY, Yu YS, Hwang JM, Kim JY, Park SS. Molecular characterization of Leber congenital amaurosis in Koreans. Molecular Vision 2008; 14:1429-36.

21. Chen Y, Zhang Q, Shen T, Xiao X, Li S, Guan L, Zhang J, Zhu Z, Yin Y, Wang P, Guo X, Wang J, Zhang Q. Comprehensive mutation analysis by whole-exome sequencing in 41 Chinese families with Leber congenital amaurosis. Invest Ophthalmol Vis Sci 2013; 54(6):4351-7.

22. Saberi M, Golchehre Z, Karamzade A, Entezam M, Eshaghkhani Y, Alavinejad E, Khojasteh Jafari H, Keramatipour M. CRB1-Related Leber Congenital Amaurosis: Reporting Novel Pathogenic Variants and a Brief Review on Mutations Spectrum. Iranian Biomedical Journal 2019; 23(5):362-8.

23. Vamos R, Kulm M, Szabo V, Ahman A, Lesch B, Schneider M, Varsanyi B, Nagy ZZ, Nemeth J, Farkas A. Leber congenital amaurosis: first genotyped Hungarian patients and report of 2 novel mutations in the CRB1 and CEP290 genes. Eur J Ophthalmol 2016; 26(1):78-84.

24. Li L, Xiao X, Li S, Jiao X, Hejtmancik JF, Zhang Q. Lack of phenotypic effect of triallelic variation in SPATA7 in a family with Leber congenital amaurosis resulting from CRB1 mutations. Molecular Vision 2011; 17:3326-32.

Page $10 / 12$ 
25. Skorczyk-Werner A, Niedziela Z, Stopa M, Krawczynski MR. Novel gene variants in Polish patients with Leber congenital amaurosis (LCA). Orphanet J Rare Dis 2020; 15(1):345.

26. den Hollander Al, Heckenlively JR, van den Born LI, de Kok YJ, van der Velde-Visser SD, Kellner U, Jurklies B, van Schooneveld MJ, Blankenagel A, Rohrschneider K, Wissinger B, Cruysberg JR, Deutman AF, Brunner HG, Apfelstedt-Sylla E, Hoyng CB, Cremers FP. Leber Congenital Amaurosis and Retinitis Pigmentosa with Coats-like Exudative Vasculopathy Are Associated with Mutations in the Crumbs Homologue 1 (CRB1) Gene. Am J Hum Genet 2001; 69:198-203.

27. Riveiro-Alvarez R, Vallespin E, Wilke R, Garcia-Sandoval B, Cantalapiedra D, Aguirre-Lamban J, Avila-Fernandez A, Gimenez A, Trujillo-Tiebas MJ, Ayuso C. Molecular analysis of ABCA4 and CRB1 genes in a Spanish family segregating both Stargardt disease and autosomal recessive retinitis pigmentosa. Molecular Vision 2008; 14:262-7.

28. Tsang SH, Burke T, Oll M, Yzer S, Lee W, Xie YA, Allikmets R. Whole exome sequencing identifies CRB1 defect in an unusual maculopathy phenotype. Ophthalmology 2014; 121(9):1773-82.

29. Khan AO, Aldahmesh MA, Abu-Safieh L, Alkuraya FS. Childhood cone-rod dystrophy with macular cystic degeneration from recessive CRB1 mutation. Ophthalmic Genet 2014; 35(3):130-7.

30. Vincent A, Ng J, Gerth-Kahlert C, Tavares E, Maynes JT, Wright T, Tiwari A, Tumber A, Li S, Hanson JV, Bahr A, MacDonald H, Bahr L, Westall C, Berger W, Cremers FP, den Hollander Al, Heon E. Biallelic Mutations in CRB1 Underlie Autosomal Recessive Familial Foveal Retinoschisis. Invest Ophthalmol Vis Sci 2016; 57(6):2637-46.

31. Ghiam BK, Wood EH, Thanos A, Randhawa S. CRB1 related retinal degeneration with novel mutation. Am J Ophthalmol Case Rep 2020; 18:100699.

32. den Hollander Al, Roepman R, Koenekoop RK, Cremers FP. Leber congenital amaurosis: genes, proteins and disease mechanisms. Prog Retin Eye Res 2008; 27(4):391-419.

33. Jalkh N, Guissart C, Chouery E, Yammine T, El Ali N, Farah HA, Megarbane A. Report of a novel mutation in CRB1 in a Lebanese family presenting retinal dystrophy. Ophthalmic Genet 2014; 35(1):57-62.

34. Bujakowska K, Audo I, Mohand-Said S, Lancelot ME, Antonio A, Germain A, Leveillard T, Letexier M, Saraiva JP, Lonjou C, Carpentier W, Sahel JA, Bhattacharya SS, Zeitz C. CRB1 mutations in inherited retinal dystrophies. Hum Mutat 2012; 33(2):306-15.

35. Izaddoost S, Nam SC, Bhat MA, Bellen HJ, Choi KW. Drosophila Crumbs is a positional cue in photoreceptor adherens junctions and rhabdomeres. Nature 2002; 416:178-83.

36. Tepass U, Theres C, Knust E. Crumbs encodes an EGF-like protein expressed on apical membranes of Drosophila epithelial cells and required for organization of epithelia. Cell 1990; 61:787-99.

37. Alves CH, Pellissier LP, Wijnholds J. The CRB1 and adherens junction complex proteins in retinal development and maintenance. Prog Retin Eye Res 2014; 40:35-52.

38. Wang H, Wang X, Zou X, Xu S, Li H, Soens ZT, Wang K, Li Y, Dong F, Chen R, Sui R. Comprehensive Molecular Diagnosis of a Large Chinese Leber Congenital Amaurosis Cohort. Invest Ophthalmol Vis Sci 2015; 56(6):3642-55.

39. Kumaran N, Moore AT, Weleber RG, Michaelides M. Leber congenital amaurosis/early-onset severe retinal dystrophy: clinical features, molecular genetics and therapeutic interventions. Br J Ophthalmol 2017; 101(9):1147-54.

40. Lotery AJ, Jacobson SG, Fishman GA, Weleber RG, Fulton AB, Namperumalsamy P, Héon E, Levin AV, Grover S, Rosenow JR, Kopp KK, Sheffield VC, Stone EM. Mutations in the CRB1 gene cause Leber congenital amaurosis. Arch Ophthalmol $2001 ; 119: 415-20$.

41. Walia S, Fishman GA, Jacobson SG, Aleman TS, Koenekoop RK, Traboulsi El, Weleber RG, Pennesi ME, Heon E, Drack A, Lam BL, Allikmets R, Stone EM. Visual acuity in patients with Leber's congenital amaurosis and early childhood-onset retinitis pigmentosa. Ophthalmology 2010; 117(6):1190-8.

42. Sharif W, Sharif Z. Leber's congenital amaurosis and the role of gene therapy in congenital retinal disorders. Int $J$ Ophthalmol 2017; 10(3):480-4.

43. Maguire AM, Simonelli F, Pierce EA, Pugh EN, Jr., Mingozzi F, Bennicelli J, Banfi S, Marshall KA, Testa F, Surace EM, Rossi S, Lyubarsky A, Arruda VR, Konkle B, Stone E, Sun J, Jacobs J, Dell'Osso L, Hertle R, Ma JX, Redmond TM, Zhu X, Hauck B, 
Zelenaia O, Shindler KS, Maguire MG, Wright JF, Volpe NJ, McDonnell JW, Auricchio A, High KA, Bennett J. Safety and efficacy of gene transfer for Leber's congenital amaurosis. N Engl J Med 2008; 358(21):2240-8.

\section{Figures}

A

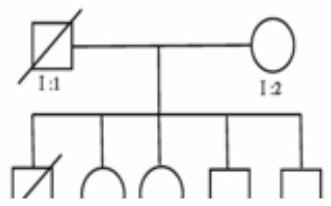

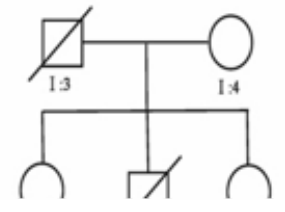

B

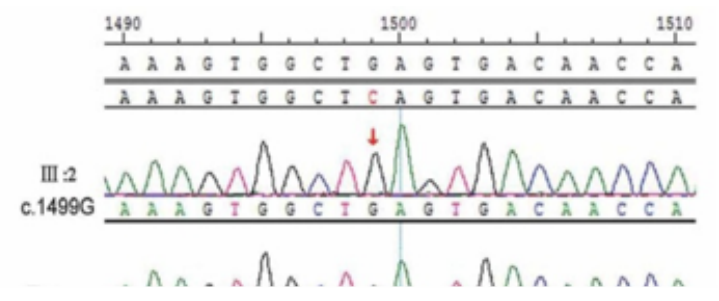

\section{Figure 1}

Pedigree of LCA family with a CRB1 variant, sequencing chromatogram, and diagnostic fundus. (A) Pedigree of LCA family with a CRB1 variant. Te proband is marked by an arrow, black symbols denote afected members, white symbols denote unafected members, squares denote males, and circles denote females. (B) Sequencing chromatograms.Affected proband show a homozygous mutation in CRB1 gene: nucleotide 1499 changed from cytosine C to guanine G (c.1499C》G) homozygous mutation, resulting in nonsense mutation of amino acids (p.S500X). (C) Diagnostic of the fundus. The proband show pigmentation of the retina at the posterior pole was peppery and salt-like, and the macular area was a mass of lesions with a lot of pigmentation.(D) Her 5 years old sister's fundus shows normal. 